\title{
The incidence of oral dryness in people over 65 years living in Lublin
}

\author{
Katarzyna Kamińska-Pikiewicz, Teresa Bachanek, Renata Cha£as*
}

Chair and Department of Conservative Dentistry and Endodontics, Medical University of Lublin, ul. Karmelicka 7, 20-081 Lublin, Poland

\section{ARTICLE INFO \\ Received 05 October 2015 \\ Accepted 20 October 2015}

\section{Keywords:}

saliva,

elderly people,

xerostomia.

\begin{abstract}
Saliva plays an important part in naturally maintaining oral homeostasis. Dry mouth or 'xerostomia', is a serious problem connected with decreased saliva secretion which considerably limits the quality of life in elderly people. The aim of the study was the assessment of the subjective oral dryness in the oral mucosa in people over 65 living in Lublin, Poland. The study was conducted among 240 people aged 65 to 96 . The patients were placed into two groups: I - 117 residents of nursing homes, II - 123 people living with their families. Assessment of the subjective oral dryness taking into account the place of residence, sex and drug administration was performed based on a questionnaire survey. In the group of nursing homes residents, $32.48 \%$ of the surveyed experienced subjective oral dryness, while among the seniors living with their families, 33.33\% reported this kind of discomfort. The conducted analysis revealed that oral dryness was more common amongst women than men, and it can be very often a side-effect of administered medicine drugs.
\end{abstract}

\section{INTRODUCTION}

Saliva plays a very important part in maintaining oral homeostasis, however, along with the aging of the organism, reverse changes occur within salivary glands which induce the impairment of their functions. Such atrophic changes are related to secretory cells being replaced by oncocytes and fatty tissue. Moreover, fibrosis can be accompanied by the adiposis of gland parenchyma. The atrophy of the minor salivary glands of the tongue and the hard palate is common in elderly people. These processes and the reduction of salivary gland vascularization, as well as the multi-morbidity countered by the administration of various medications, is characteristic for elderly people, and inevitably leads to the decrease in the production of saliva, and, hence, reduced enzyme and defense activity [1].

Dryness of the mouth, or 'xerostomia', is a serious problem connected with decreased saliva secretion and considerably limits the quality of life in elderly people $[5,6,12]$. Two forms of this condition exist:

1. True xerostomia - primary (xerostomia vera): caused by the impairment of salivary gland function as a symptom of local or systemic disease.

\footnotetext{
* Corresponding author

e-mail: renata.chalas@umlub.pl
}

2. Symptomatic xerostomia - secondary/false (xerostomia spuria): induced by medications, or changes in emotional states, but without changes in the conditional state of the salivary glands.

Commonly, dryness of the mouth is naturally experienced around the age of 50-70, and affects at least $30-40 \%$ of all elderly people [19]. It frequently accompanies menopause in women or occurs post-menopause. Xerostomia may result in taste disorders, pain while eating irritating foods or drink items (such as fruit or alcohol), difficulties with chewing and swallowing (which lead to the loss of appetite), the eating of less nutritious, pulpy food, as well as malnutrition. It also brings about difficulty in speaking. Furthermore, a decrease in the amount of the secreted saliva reduces natural oral cleaning mechanisms contributing to the accumulation of food debris, shredded epithelial cells and bacterial plaque. This state promotes the development of crown and root dental caries - particularly in situations of poor oral hygiene. Hence, defects of non-carious origin, especially erosion, frequently occur. Xerostomia may also contribute to bad breath (halitosis). Moreover, dryness of the oral mucosa promotes the development of fissures, injuries, ulcerations, atrophic mucositis, angular cheilitis, and exfoliative cheilitis. In addition, it may cause burning sensation, as well as a coated and wrinkled tongue. Xerostomia often 
leads to catarrhal gingivitis and secondary bacterial and viral infections. Plus the resulting acidic $\mathrm{pH}$ promotes an increase in the number of Streptococcus mutans and Lactobacillus. The condition, furthermore, substantially impedes adaptation and the use of removable dentures. Finally, in patients afflicted with xerostomia, the symptoms of stomatitis prothetica in the form of ulcers and abrasions are observed more frequently than in patients with no saliva secretion disturbances $[5,14,19]$.

Possible causes of oral dryness include systemic and local reasons, as well as the side effects of drugs taken (pharmacological xerostomia) [7]. Pharmacological xerostomia is a serious problem, since there are about 400 currently registered medications that contribute to oral dryness. Elderly people more frequently suffer from several chronic diseases which results in the use of various medications. Seniors, beside their prescribed drugs, also commonly self-administer non-prescription ones without consultation with their doctor. Pharmaceutical preparations that induce decreasing saliva secretion include: antihistamines, antidepressants, sedatives, anticholinergics, anti-arrhythmics, antihypertensives, hormonal drugs, diuretics, narcotics, appetite suppressants, bronchodilators, antineoplastics, chemotherapeutics, antispasmodics, cytostatics, immunostimulants, as well as analgesics and psychotropic medications. Xerostomia is also brought about by the radiotherapy employed in the treatment of neoplasms of the facial skeleton and neck. A limitation of the amount of taken drugs improves saliva secretion, although it is not always possible because of patient's general health state $[2,5,6,19]$.

\section{AIM OF THE STUDY}

The aim of the study was to assess the degree of subjective dryness in the oral mucosa, in people over 65 who are residing in Lublin, Poland.

\section{MATERIAL AND METHODS}

The study was conducted in Lublin, a city situated in the south-east part of Poland. Public care home residents formed one part of the study-population. A total of 117 individuals (57 males and 60 females) were included in this group. The other part of the study-population, was a comparative group of individuals living at home, but who were out-patients of the Dental Clinical Center, Medical University of Lublin. This group consisted of 123 individuals (63 men and 60 women). The inclusion criteria for both groups were age 65 years and above and no signs of acute illness being seen at the time of the examination. All patients were comfortable in their immediate environment and met the minimal physical and mental criteria so as to give informed consent for participation in the study. The study tool was a survey questionnaire (residence, gender, prescribed and non-prescribed drug use, as well as assessment of the subjective sensation of oral dryness). The mean age of the nursing home resident patients was $75.91 \pm 7.50$ years, and that of the walk-in (home) patients: $73.69 \pm 6.10$ years.

The study protocol was reviewed and fully approved by the Bioethics Committee of the Medical University of Lublin, Poland. The obtained results were statistically analyzed and a chi-square test was performed to evaluate the differences. The results were considered significant at $\mathrm{p}<0.05$.

\section{RESULTS}

The conducted study has revealed that in the group of nursing home residents, $32.48 \%$ of the surveyed had the sensation of oral dryness. Among the home residents, 33.33\% people reported subjective dryness in the oral cavity (Table 1). In both groups, more female participants reported a selfassessment of oral-dryness than did males, but statistical analysis revealed an essential correlation between oral dryness and sex in the studied groups only amongst the nursing home residents: $45.00 \%$ of all women and $19.30 \%$ of all men stated a self-assessment of dryness, $\mathrm{p}=0.003$ (Table 2). No statistically significant correlation was found between oral dryness and sex in the home residents: $38.33 \%$ of all women and $28.57 \%$ of all men reported oral dryness, $\mathrm{p}=0.25$ (Table 3 ).

Table 1. Residency and incidence of oral dryness in the examined groups

\begin{tabular}{|l|c|c|c|c|}
\hline \multirow{2}{*}{ Oral symptom } & \multicolumn{2}{|c|}{ Nursing home } & \multicolumn{2}{c|}{ Family home } \\
\cline { 2 - 5 } & $\begin{array}{c}\text { Number of } \\
\text { persons }\end{array}$ & $\%$ & $\begin{array}{c}\text { Number of } \\
\text { persons }\end{array}$ & $\%$ \\
\hline Dryness & 38 & $32.48 \%$ & 41 & $33.33 \%$ \\
\hline
\end{tabular}

Table 2. Nursing home residency: Incidence of oral dryness and sex

\begin{tabular}{|l|c|c|c|}
\hline \multirow{2}{*}{ Sex } & No & Yes & Total \\
\cline { 2 - 4 } & \multicolumn{3}{|c|}{$\begin{array}{c}\text { Number of persons } \\
\%\end{array}$} \\
\hline \multirow{3}{*}{ Women } & 33 & 27 & 60 \\
\hline \multirow{3}{*}{ Men } & $55.00 \%$ & $45.00 \%$ & $100.00 \%$ \\
\hline \multirow{3}{*}{ Total } & 46 & 11 & 57 \\
\cline { 2 - 4 } & $80.70 \%$ & $19.30 \%$ & $100.00 \%$ \\
\hline \multicolumn{4}{|c|}{ Statistical analysis: Chi2 $=8,80 ; \mathrm{p}=0,003 *$} \\
\cline { 2 - 4 } & 79 & 38 & 117 \\
\hline
\end{tabular}

Table 3. Family home residency: Incidence of oral dryness and sex

\begin{tabular}{|c|c|c|c|}
\hline \multirow{2}{*}{ Sex } & No & Yes & Total \\
\hline & \multicolumn{3}{|c|}{$\begin{array}{l}\text { Number of persons } \\
\%\end{array}$} \\
\hline \multirow{2}{*}{ Women } & 37 & 23 & 60 \\
\hline & $61.67 \%$ & $38.33 \%$ & $100.00 \%$ \\
\hline \multirow{2}{*}{ Men } & 45 & 18 & 63 \\
\hline & $71.43 \%$ & $28.57 \%$ & $100.00 \%$ \\
\hline \multirow{2}{*}{ Total } & 82 & 41 & 123 \\
\hline & $66.67 \%$ & $33.33 \%$ & $100.00 \%$ \\
\hline
\end{tabular}

The conducted studies indicated that among all participants who reported oral dryness, $72.16 \%$ of the surveyed were taking medications which could induce oral dryness as a side effect. Of the remaining, $22.78 \%$ of all participants were taking medications which did not induce oral dryness, while $5.06 \%$ reported that they were not taking any medications at all. From the group of medications bringing about 
perceived xerostomia, the respondents who complained of the sensation of oral dryness most frequently stated a use of antihypertensives $-27.85 \%$, sedatives $-17.72 \%$, bronchodilators $-15.19 \%$, psychotropics $-12.66 \%$, anti-dizziness medicines $-12.66 \%$, and less frequently stated a use of diuretics $-8.86 \%$, antidepressants $-7.59 \%$, anti-arrhythmics $-6.33 \%$, analgesics $-2.53 \%$, anti-Parkinson's drugs $-2.53 \%$, immunostimulants $-1.27 \%$, or other medicaments $-1.27 \%$ (Table 4 ).

Table 4. Reported medications: Individuals experiencing dryness of the oral mucosa

\begin{tabular}{|l|c|c|}
\hline \multicolumn{1}{|c|}{ Types of Medications } & Number of people & $\%$ \\
\hline antihypertensive & 22 & $27.85 \%$ \\
\hline other medications not causing dryness & 18 & $22.78 \%$ \\
\hline sedatives & 14 & $17.72 \%$ \\
\hline bronchodilators & 12 & $15.19 \%$ \\
\hline psychotropic & 10 & $12.66 \%$ \\
\hline anti-dizziness medications & 10 & $12.66 \%$ \\
\hline diuretics & 7 & $8.86 \%$ \\
\hline antidepressants & 6 & $7.59 \%$ \\
\hline anti-arrhythmic & 5 & $6.33 \%$ \\
\hline do not take medications & 4 & $5.06 \%$ \\
\hline analgesics & 2 & $2.53 \%$ \\
\hline anti-Parkinson's medications & 2 & $2.53 \%$ \\
\hline immunostimulants & 1 & $1.27 \%$ \\
\hline others & & $1.27 \%$ \\
\hline
\end{tabular}

\section{DISCUSSION}

Elderly people commonly are afflicted with disorders of saliva secretion. The decreased secretion leads to an increase of oral mucosa sensitivity to mechanical and chemical stimuli manifesting in the sensation of oral dryness and burning [14]. In our work, we saw that $33.48 \%$ of all nursing home residents in Lublin of both sexes who took part in the study reported dryness (women $-45.00 \%$, men $-19.30 \%$ ). For a comparative study in Warsaw, this figure was twice less the percentage $-14 \%$, and the sensation of dryness was reported only by women [20]. The sensation of oral dryness was also reported among residents of nursing home residents in greater Poznan [8]. A lower percentage of people (both nursing home residents and home residents, $6 \%$ and $2 \%$, respectively) reported the sensation of dryness and burning in the oral cavity in a comparative study in Germany[3]. However, a comparative degree of oral dryness sensation to that of our Lublin study was seen in a similar study of nursing home residents in Brazil (Taubate) - 36.3\% [10] and in England (West Hertfordshire) - 35\% [17], whereas a higher percentage of senior nursing home residents in Iran reported this effect $-42.1 \%$ [16]. Moreover, among senior nursing home residents in Sweden, the subjective sensation of dryness was stated as being experienced by $41 \%-78 \%$ of the surveyed (depending on criteria used), whereas the sensation of subjective dryness was noted in $44 \%$ of the total population [4].

In our own study, the sensation of dryness was noted in $33.33 \%$ of seniors participating in the study who resided at home, and no significant correlation was noted between oral dryness and sex. A comparative study in Bytom generated the figure of $10 \%$ [9], and that consisting of seniors of the University of Third Age in Wroclaw - 10\% (dryness only) [15]. Furthermore, about $10 \%$ of all seniors prescribed dentures and who were out-patients of the Department of Dental Prosthetics of the Medical University in Wroclaw [13] complained of oral mucosa pain and burning sensation, as did $42.5 \%$ of a similar group of patients in Warsaw [11]. Among senior patients from Gdansk, 51\% reported the incidence of periodic disorders of the oral mucosa such as burning, soreness, stabbing, itching and oversensitivity to stimuli [18]. Regarding place of residence, whether in nursing home or own home, we observed that the subjective sensation of oral dryness occurred at a similar percentage. This percentage was generally higher than that of the data presented by other authors. What is more, no explicit influence of social and living conditions on the incidence of xerostomia was noted. However, it was observed that the majority of people complaining of the dryness sensation were taking medicines which bring about a decrease in saliva secretion.

Old age promotes the development of xerostomia and this negatively affects quality of life. Therefore, it is fundamental to draw elderly people's attention towards the necessity of following the rules of proper oral hygiene, drinking sufficient amounts of sugar-free fluids, stimulating saliva e.g. by chewing sugar-free gum, as well as practicing healthy dietary habits. It is advisable that seniors avoid taking in spicy, salty food, very hot or cold drinks, fresh fruit juices, coffee, black tea, simple sugars, alcohol and tobacco. Finally, in seniors with diagnosed xerostomia, it is recommended that they be prescribed saliva replacement preparations or systemic medications $[5,19]$.

\section{CONCLUSION}

In the group of elderly people living in Lublin, whether residing in nursing homes or at home, oral dryness is experienced by women more than men, and it is most often a sideeffect of administered or self-administered pharmaceuticals.

\section{REFERENCES}

1. Bourdiol P., Mioche L., Monier S.: Effect of age on salivary flow obtained under feeding and non-feeding conditions. J. Oral Rehab., 31, 445, 2004.

2. De Barros Pontes C., Polizello A.C.M., Spadaro A.C.C.: Clinical and biochemical evaluation of the saliva of patients with xerostomia induced by radiotherapy. Braz. Oral Res., 18, 69, 2004.

3. Frączak B., Lupa-Bühmann J., Sobolewska E.: Prosthetic treatment needs among the elder in Germany. Ann. Acad. Med. Stetin., 53, 134, 2007.

4. Gerdin E.W. et al.: Impact of dry mouth conditions on oral healthrelated quality of life in older people. Gerodontology, 22, 219, 2005.

5. Gil-Montoya J.A., Guardia-López I., González-Moles M.A.: Evaluation of the clinical efficacy of a mouthwash and oral gel containing the antimicrobial proteins lactoperoxidase, lysozyme and lactoferrin in elderly patients with dry mouth - a pilot study. Gerodontology, 25, 3, 2008.

6. Hugo F.N. et al.: Association of chronic stress, depression symptoms and cortisol with low saliva flow in a sample of south-Brazilians aged 50 years and older. Gerodontology, 25, 18, 2008. 
7. Ikebe K. et al.: Impact of dry mouth and hyposalivation on oral health-related quality of life of elderly Japanese. Oral Surg. Oral Med. Oral Path. Oral Radiol. Endo., 103, 216, 2007.

8. Koczorowski R., Gawriołek M., Siniawska J.: Prosthetic needs of nursing home residents in Wielkopolska. Dent. Med. Probl., 45, 425, 2008.

9. Łata A., Monkos-Jaremczuk E., Staroń K.: The actual estimation of the frequency of accurence of prosthetic stomatopathy and its evolution favourable factors. Poradnik Stomatol., 2, 5, 2005.

10. Marchini L. et al.: Self-reported oral hygiene habits among institutionalised elderly and their relationship to the condition of oral tissues in Taubaté, Brazil. Gerodontology, 23, 33, 2006.

11. Nawrocka-Furmanek J. et al.: A survey of the results of prosthetic rehabilitation in a group of geriatric patients with removable dentures". Nowa Stomat., 3, 119, 2006.

12. Ogasawara T. et al.: Potential factors responsible for dryness of the dorsum of the tongue in elderly requiring care. Gerodontology, 25, 217, 2008.

13. Panek H. et al.: Analysis of complaints reported by patients in their prosthetic past. Dent. Med. Probl., 41, 3, 489, 2004.
14. 1Pogorzelska-Stronczak B., Bubiłek-Bogacz A., Szporek B.: Selected stomatological problems in elderly. Wiad. Lek., 54, 88, 2001.

15. Pregiel B. et al..: Condition of oral mucous membrane of members of audience attending University for Third Age in Wroclaw. Mag. Stomat., 11, 72, 2006.

16. Rabiei M. et al.: Prevalence of oral and dental disorders in institutionalised elderly people in Rasht, Iran. Gerodontology, 27, $174,2010$.

17. Simons D. et al.: Relationship between oral hygiene practices and oral status in dentate elderly people living in residential homes. Comm. Dent. Oral Epidem., 29, 464, 2001.

18. Słomińska M. et al.: Analysis of questionnaires among denture wearers. Prosthodontics, 50, 19, 2000.

19. Turner M.D., Ship J.A.: Dry mouth and its effects on the oral health of elderly people. J. Am. Dent. Assoc., 138, 15S, 2007.

20. Woś A. et al.: Evaluation of the frequency of changes on the tongue in elderly people in relation to sex, systemic diseases and usage of dentures. Nowa Stomatol., 3, 128, 2006. 